\title{
Mach 10 Stage Separation Analysis for the X43-A
}

\author{
Paul V. Tartabini* \\ NASA Langley Research Center, Hampton, VA, 23681 \\ David M. Bose ${ }^{\dagger}$, Mark N. Thornblom ${ }^{\ddagger}$ and J.P. Lien ${ }^{\ddagger}$ \\ Analytical Mechanics Associates, Inc, Hampton, VA, 23666 \\ and \\ John G. Martin ${ }^{\S}$ \\ NASA Langley Research Center, Hampton, VA, 23681
}

This paper describes the pre-flight stage separation analysis that was conducted in support of the final flight of the X-43A. In that flight, which occurred less than eight months after the successful Mach 7 flight, the X-43A Research Vehicle attained a peak speed of Mach 9.6. Details are provided on how the lessons learned from the Mach 7 flight affected separ ation modeling and how adjustments were made to account for the increased flight Mach number. Also, the procedure for defining the feedback loop closure and feed-forward parameters employed in the separation control logic are described, and their effect on separation performance is explained. In addition, the range and nominal values of these parameters, which were included in the Mission Data Load, are presented. Once updates were made, the nominal pre-flight trajectory and Monte Carlo statistical results were determined and stress tests were performed to ensure system robustness. During flight the vehicle performed within the uncertainty bounds predicted in the pre-flight analysis and ultimately set the world record for airbreathing powered flight.

\section{Nomenclature}

$\begin{array}{ll}\alpha & =\text { Angle of attack, deg } \\ \beta & =\text { Sideslip angle, deg } \\ \beta_{\max } & =\text { Peak sideslip angle, deg } \\ C_{A} & =\text { Axial force coefficient } \\ C_{N} & =\text { Normal force coefficient } \\ C_{m} & =\text { Pitching moment coefficient } \\ \delta_{\text {elev }} & =\text { Elevator deflection, deg } \\ \delta_{\text {rud }} & =\text { Rudder deflection, deg } \\ d \alpha / d t & =\text { Rate of change of angle-of-attack, deg } / \mathrm{s} \\ \phi & =\text { Body roll angle, deg } \\ F x & =\text { Axial Force in vehicle body frame, } \mathrm{lb}_{\mathrm{f}} \\ p & =\text { Body roll rate, deg/s } \\ q & =\text { Body pitch rate, deg } / \mathrm{s} \\ r & =\text { Body yaw rate, deg/s } \\ \sigma & =\text { standard deviation } \\ X_{S E P} & =\text { Horizontal separation distance, inches }\end{array}$

\footnotetext{
* Aerospace Engineer, Vehicle Analysis Branch, M/S 451.

Aerospace En gineer, 303 Butler Farm Rd Suite 104A, Member, AIAA.

\# Aerospace Engineer, 303 Butler Farm Rd Suite 104A.

${ }^{\S}$ Senior Aerospace Engineer, Vehicle Analysis Branch, M/S 451, Senior Member, AIAA.
} 


\section{Introduction}

$\mathrm{O}$ November 16, 2004 NASA's X-43A demonstrator vehicle, the first free flying air-breathing scramjet powered aircraft, successfully completed its final test flight, reaching Mach 9.6 - a world speed record for powered flight. This flight occurred less than 8 months after the X-43A's first successful flight, in which the planned peak speed of Mach 7 was attained. ${ }^{1-2}$

One of the highest risk events during both of these flights was the separation of the X-43A research vehicle (RV) from the Hyper-X Launch Vehicle (HXLV). Although stage separation of two non-axisymmetric bodies at these flight conditions (Mach 7 and 10, dynamic pressure of 1000 psf) had never been done, in both instances stage separation was successful and the RV reached its target attitude prior to the start of the test sequence without recontacting the HXLV.

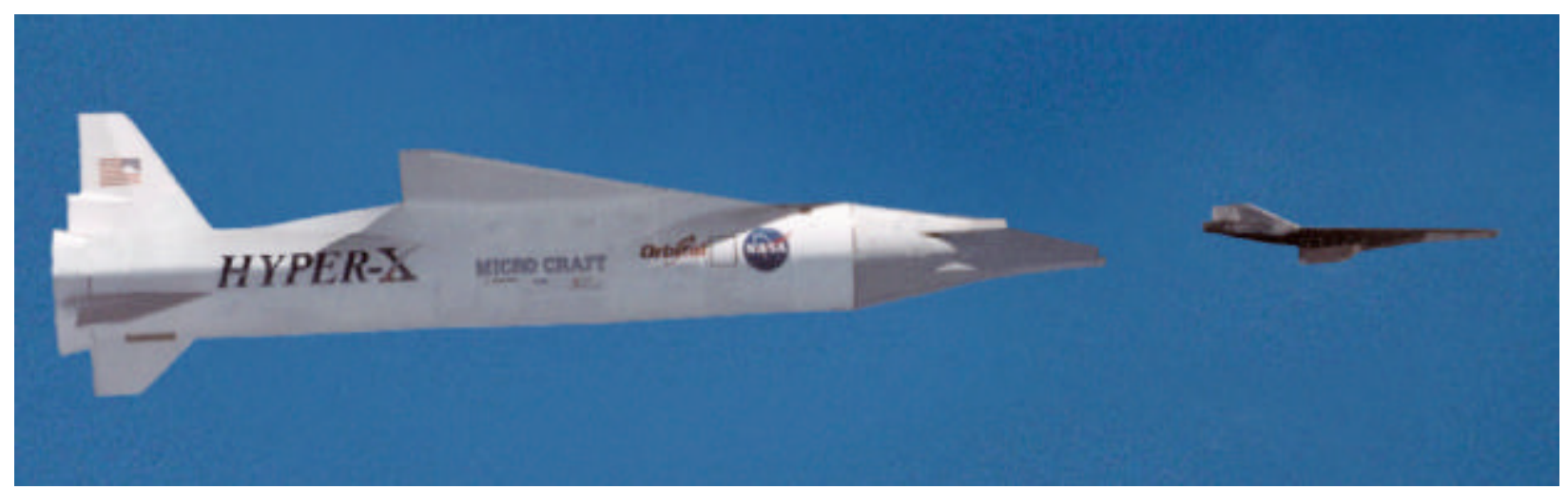

Figure 1. Artist concept of X-43A stage separation.

Even though the success of the stage separation in the first X-43A Mach 7 flight increased the overall confidence in the stage separation simulation modeling, there were some minor differences that were observed between the flight data and pre-flight models. Efforts were made to understand these differences by systematically reconciling the simulation models with the flight data and incorporating the relevant findings and model adjustments into the Mach 10 separation simulation. In addition, these flight data comparisons were used to reassess model uncertainty levels and define additional stress cases that were run to ensure system robustness. Finally, since the vehicle had less stability and control effectiveness at Mach 10, both Computational Fluid Dynamics (CFD) and wind tunnel results were used to modify the aerodynamic interference model to better match the Mach 10 flight conditions. The changes in the vehicle aerodynamics were significant enough to require that the separation control logic be redesigned. This paper describes the development of the overall Mach 10 separation strategy and the corresponding risk reduction activities that were undertaken to account for the different flight condition and demonstrate that the system was robust to any errors seen in the previous Mach 7 flight.

\section{Mach 10 Separation Simulation Development}

\section{A. Simulation Methodology}

To assess the risk associated with stage separation, a 14 degree-of-freedom simulation had been created early in the X-43A program using the Automatic Dynamic Analysis of Mechanics Systems (ADAMS) Simulation software. ${ }^{3}$ This simulation was composed of various user-defined modules that modeled the vehicle aerodynamics, ejector pistons, control system, inertial navigation system, and control surface actuators. The entire simulation, which includes the user-defined modules wrapped around the ADAMS solver, is known as SepSim. ${ }^{7}$ In addition, another independent separation simulation was developed prior to the Mach 7 flight as an additional risk reduction measure to verify the SepSim results. This independent simulation, which was developed using the Program to Optimize Simulated Trajectories (POST) ${ }^{4,5}$, was less detailed than SepSim, but was simpler, more concise and ran faster, while providing similar results. ${ }^{6}$

In order to take advantage of the strengths of each of these simulations, the Mach 10 updates were applied to both POST and SepSim. Because of its rapid Monte Carlo capability and efficiency as a parametric analysis tool, POST was used as the "work-horse" simulation while the higher fidelity SepSim, which, unlike the POST simulation, included high-order actuator and sensor models, served as the final truth model. Each simulation was verified 
separately using standalone models. In addition, both nominal and off-nominal cases were run in both POST and SepSim and the results were compared and shown to be in good agreement. Similar comparisons were made periodically throughout the Mach 10 study to ensure that traceability between the two simulations was maintained.

\section{B. Mach 10 Separation Timeline}

The entire Mach 10 stage separation event was $2.5 \mathrm{sec}$ in duration. The nominal separation scenario is shown in Fig. 2. The event begins as two pyrotechnically actuated pistons push the vehicles apart. At $100 \mathrm{~ms}$ the pistons reach the end of their 9-inch extensions and the HXLV commands a 10 deg elevon deflection to induce a nose-down pitching motion, moving it further away from the separated RV as it descends uncontrolled back to Earth. For roughly the first $250 \mathrm{~ms}$ the two vehicles are in close enough proximity that aerodynamic interference effects have a significant effect on the trajectory. These effects gradually diminish and are gone by $500 \mathrm{~ms}$ when the horizontal separation distance between the vehicles is approximately $110 \mathrm{in.} \mathrm{Figure} 2$ shows the times of the various loop closures with rate feedback loops closing at the end of the piston push and outer loops $(\alpha$ and $\phi)$ closing at the beginning of free flight. The yaw rate loop is faded in to improve off-nominal performance by balancing the likelihood of sideslip excursions and high rudder actuator rates.

The primary objective was to maximize the probability of a successful separation. There were three specific requirements that were the primary means of defining separation success: 1) to avoid a re-contact between the two flight vehicles, 2) to minimize the likelihood of a loss of control resulting in large attitude excursions (i.e., $\alpha$ or $\beta$ exceeding $\pm 10^{\circ}$ ), and 3 ) to reach the attitude target box required for the engine test at the end of the 2.5 sec separation event $\left(\alpha=1.0^{\circ} \pm 1.0^{\circ}, \beta=0.0^{\circ} \pm 0.5^{\circ}\right)$ with zero attitude rates.

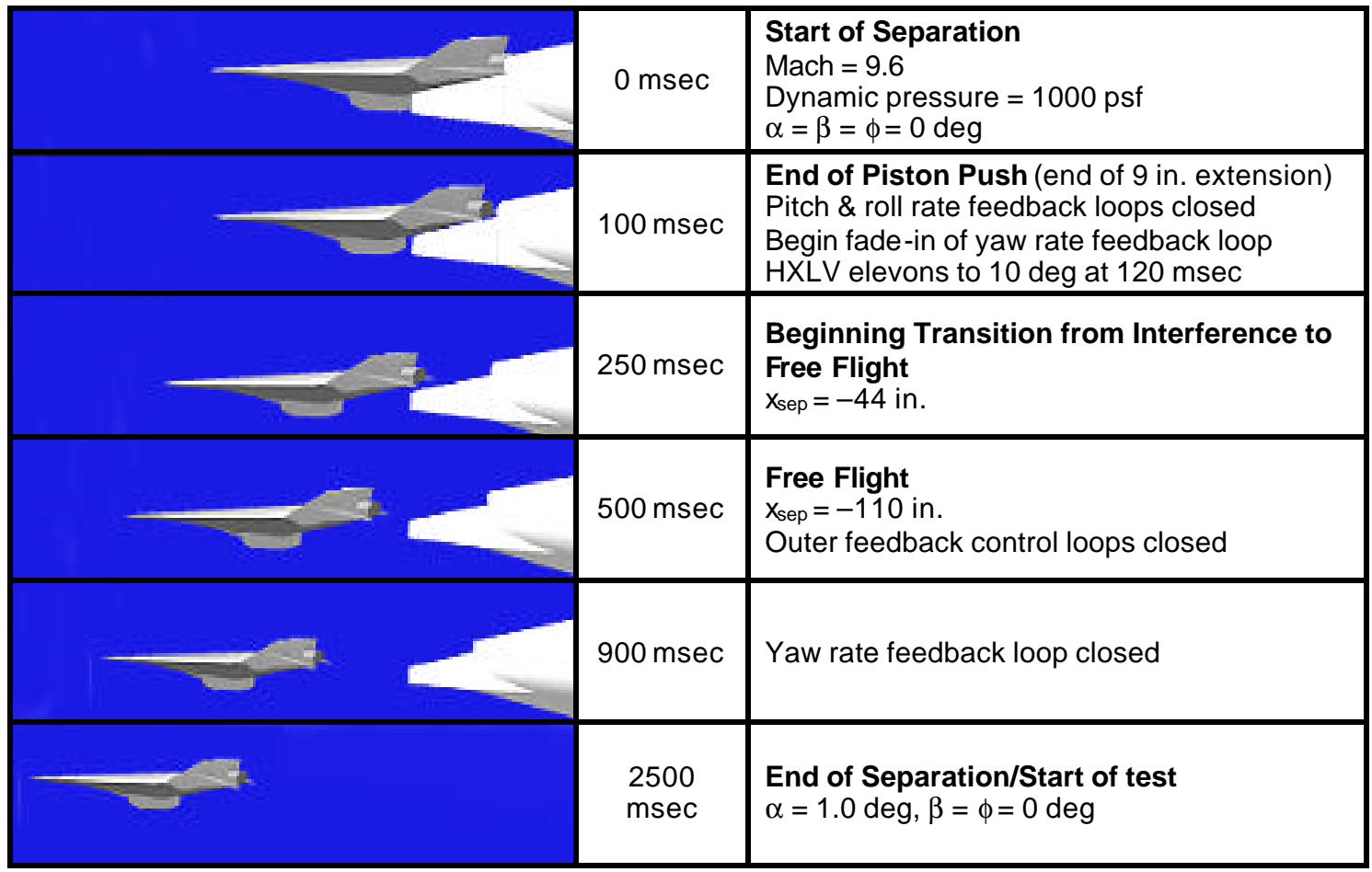

Figure 2. Nominal Mach 10 separation ti meline.

\section{Simulation Model Updates}

In general, the structure of the Mach 10 stage separation simulation was the same as that used to conduct the preflight analysis for the Mach 7 flight; however, a number of updates had to be made to account for the different vehicles and flight condition. In addition, model reconciliation studies were conducted in which key simulation models were compared with Mach 7 flight data to assess how well the flight data was captured by the pre-flight Monte Carlo uncertainty bounds and, if necessary, determine how to adjust model parameters to best fit the observed response. 
The separation conditions shown in Table 1 were similar to the Mach 7 conditions with the exception of the separation Mach number. As with the Mach 7 pre -flight Monte Carlo analysis, extremely conservative uncertainty models were assumed. For instance, even though the HXLV was predicted to deliver the RV to a separation angleof-attack between -0.6 and $1.0 \mathrm{deg}$, this uncertainty was assumed to be as high as $\pm 1.5 \mathrm{deg}$. A similar conservative approach was taken with the other initial separation condition uncertainties listed in Table 1.

Table 1. Mach 10 separation conditions and uncertainty.

\begin{tabular}{lcc}
\hline \hline \multicolumn{1}{c}{ Separation Condition } & Mean & $\mathbf{\pm 3}-\boldsymbol{\sigma}$ \\
\hline Dynamic Pressure, psf & 1066 & 210 \\
Mach Number & 9.6 & 0.6 \\
Flight Path Angle, deg & 1.5 & 1.0 \\
Angle-of-attack, deg & 0.0 & 1.5 \\
Sideslip Angle, deg & 0.0 & 1.0 \\
Body Roll Angle, deg & 0.0 & 15.0 \\
Body Pitch Rate, deg/s & -0.18 & 3.0 \\
Body Yaw Rate, deg/s & 0.0 & 3.0 \\
Body Roll Rate, deg/s & 0.0 & 6.0 \\
\hline
\end{tabular}

A number of mass property updates were required to account for differences in both vehicles with respect to the Mach 7 flight. The largest change was due to the fact that the HXLV did not need to carry ballast to reach the Mach 10 separation conditions. Consequently, at separation the HXLV mass was lower and there was a significant shift in $\mathrm{cg}$ position. The nominal weight of the entire HXLV/RV stack at the initiation of stage separation was $10,415 \mathrm{lb}$ with the RV alone comprising $2,823 \mathrm{lb}$ of the total.

The locations and force profiles of the ejector pistons were the same as in the Mach 7 flight. As described in Reference 6 , the separation process was initiated by two pyrotechnically activated ejector pistons that emanated from the HXLV and remained in contact with the RV for approximately $0.1 \mathrm{sec}$ until reaching the end of their 9-in. stroke length. The pistons were modeled in the simulation as a spring with a test-derived axial force profile that was a function of time and extension length, and with lateral restoring forces computed from beam deflection theory. After the Mach 7 flight the piston model was assessed by comparing the axial acceleration from simulation results to acceleration data derived from telemetry. The flight data was well captured in the simulation results as illustrated in Fig. 3. Additional comparisons were performed that indicated the piston extension time histories were also consistent with simulation predictions. As a result of this agreement, no modifications were made to the simulation piston models.

The aerodynamic model employed in the separation simulation was a complex database constructed from wind tunnel data and computational fluid dynamic calculations that provided force and moment coefficients for three different vehicle configurations (stack, HXLV and RV) in regions of free-flight and aerodynamic interference. ${ }^{8-10}$ Two different eightdimensional databases were used to compute the interference aerodynamics on each vehicle depending on the value of $X_{S E P}$ (axial separation distance). The vehicles were assumed to be completely in the interference region from separation until $\mathrm{X}_{\mathrm{SEP}}=-44$ in (note that $\mathrm{X}_{\mathrm{SEP}}$ is initially 0 when separation commences and becomes more negative as the vehicles move apart). In the Mach 7 analysis, when $\mathrm{X}_{\mathrm{SEP}}$ reached -69 inches the vehicles were assumed to be out of the interference region and a different free flight database was used to compute the

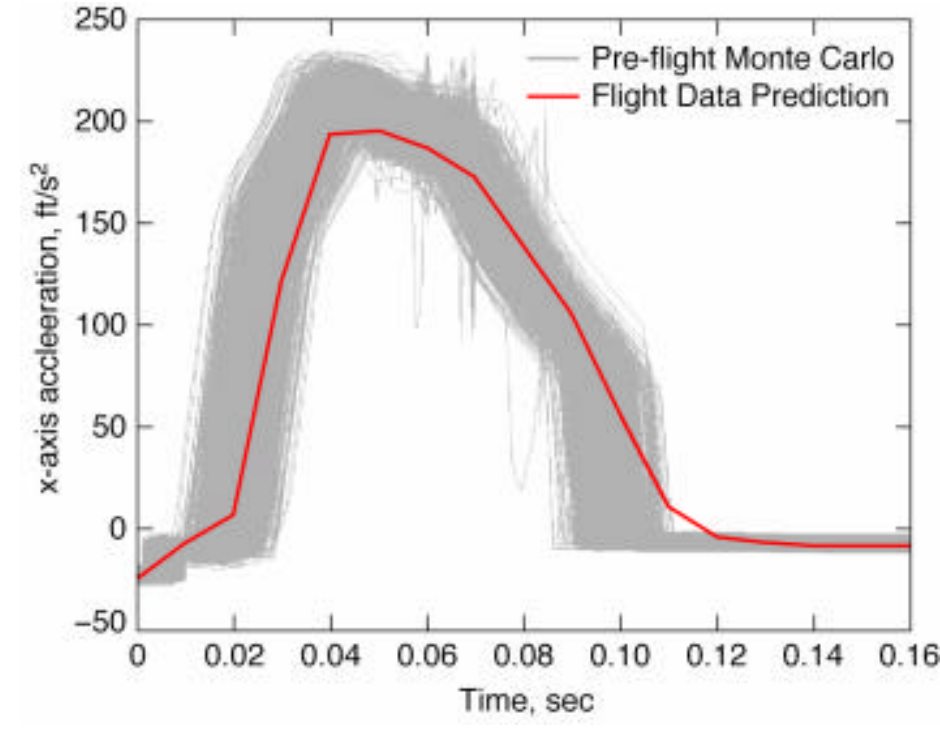

Figure 3. Mach 7 axial acceleration profile during piston firing. flight data vs. pre-flight Monte Carlo. 
aerodynamic forces and moments. In the region between - 44 and -69 inches, the base coefficients were ramped linearly from the interference values to the free flight values. The aerodynamic coefficient build-up in the simulation also included Monte Carlo uncertainty terms to account for potential errors in the model. In the interference region, the uncertainty of each coefficient was modeled as an additive increment that was a function of $\mathrm{X}_{\mathrm{SEP}}$. In the free flight region, a multiplier was applied to each nominal coefficient and an incremental bias term was also added.

Even though the Mach 7 flight had been conducted without any major problems during separation and the RV achieved an acceptable attitude prior to the initiation of the engine test sequence, the vehicle transitioned to the commanded angle-of-attack slower than what had been predicted in pre-flight simulations (see Fig. 4). Moreover, the response was outside of the pre-flight Monte Carlo predictions.

To better understand these discrepancies, a systematic model reconciliation study was undertaken to determine the value of the aerodynamic uncertainty terms that best aligned the simulation predictions with the observed flight data. These uncertainty values were adjusted iteratively until the angle of attack, angle of sideslip, and body roll angle from the simulation results matched the flight response. Additionally, an iterative Kalman filter was developed to estimate the uncertainty terms using aerodynamic forces and moments derived from flight accelerations. The main advantage of the Kalman filter is that it had the ability to estimate the parameters simultaneously. These two methods were used together to understand why the profiles differed and how the mo dels could have been adjusted so that the Monte Carlo predictions better captured the flight behavior.

Figure 4 illustrates that the aerodynamic uncertainty terms could be adjusted so that the simulation results matched the flight data reasonably well. These results confirmed that the slowerthan-predicted angle-of-attack response was due to inaccuracies in the prediction of the pitch trim location. While the aerodynamic uncertainty model that was employed in the simulation had adequately captured uncertainties in the magnitude of the aerodynamic coefficients (i.e., slope of the pitching moment curve), the estimated variation in the pitch trim location was too low because the $\mathrm{Cm}_{0}$ bias term had been under predicted. Even so, the integrator gain was sufficient so that the controller was able to completely iemove the angle-of-attack error prior to the start of the engine test sequence.

Despite this result, the $\mathrm{Cm}_{0}$ uncertainty levels were not changed for the Mach 10 simulation analysis because CFD and wind tunnel results did not conclusively show that a similar behavior would occur at the higher Mach number. Instead,

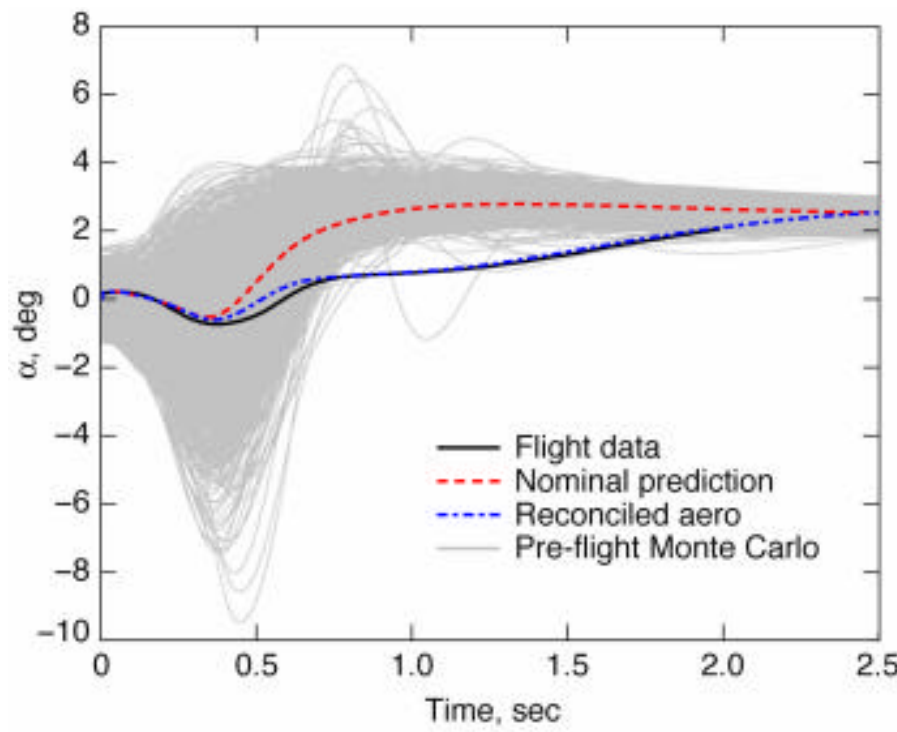

Figure 4. Comparison of Mach $7 \mathrm{RV}$ angle-of-attack time history. Flight data vs. pre-flight Monte Carlo.

to allow for the possibility of similar underprediction of $\mathrm{Cm}_{0}$ bias at Mach 10, the controller was modified by increasing the integrator gain for angle -of-attack. A stress test was conducted using the updated simulation with a conservative $\mathrm{Cm}_{0}$ uncertainty range to show that the system was robust to the differences that were observed in Mach 7 flight. The results of this stress test are discussed in a later section.

Another result of the aerodynamic model reconciliation study came when using the Kalman filter to estimate the free flight aerodynamic uncertainty parameters. Recall that in the Mach 7 simulation aerodynamic interference effects were assumed to be completely dissipated at a horizontal separation distance of 69 inches. When the Kalman filter was used to estimate the free flight uncertainty model parameters to best match the aerodynamic force and moment flight data just outside this region, at an $\mathrm{X}_{\mathrm{SEP}}$ value of -75 inches, the filter was unable to converge. This process was repeated for a range of $\mathrm{X}_{\mathrm{SEP}}$ values, and the results for the axial force are shown in Fig. 5. The plot shows that the filter began to converge with the flight data around $X_{\mathrm{SEP}}=-110$ inches, suggesting that aerodynamic interference effects actually persisted until then. Consequently, the region of transition from aerodynamic interfe rence to free flight was increased in the Mach 10 simulation; that is, the point at which the free flight aerodynamic database was used alone was extended to an $\mathrm{X}_{\mathrm{SEP}}$ value of -110 inches. In addition, a $3-\sigma$ uncertainty value of \pm 10 inches was included for this parameter in Monte Carlo analyses. 
The aerodynamic interference database (used exclusively in the first 44 inches of horizontal separation distance) was predominantly constructed using wind tunnel measurements taken at the Arnold Engineering Development Center at a Mach 6 test condition. This data was used in the Mach 7 pre-flight analysis without a Mach 6-to-Mach 7 adjustment. For the Mach 10 flight, the longitudinal coefficients $\mathrm{C}_{\mathrm{A}}, \mathrm{C}_{\mathrm{N}}$ and $\mathrm{C}_{\mathrm{m}}$ were adjusted to account for the difference in Mach by adding a constant bias that was based on Mach 10 CFD solutions. The increments that were applied were $\Delta \mathrm{C}_{\mathrm{A}}=$ $+0.0023, \Delta \mathrm{C}_{\mathrm{N}}=+0.0062$ and $\Delta \mathrm{C}_{\mathrm{m}}=-0.00075$. Figure 6 shows the nose-down effect of the adjustment on the pitching moment coefficient in the first 44 inches of $X_{S E P}$ for a nominal separation trajectory. In the simulation, these biases were linearly ramped to zero between $\mathrm{X}_{\mathrm{SEP}}=-44$ and -110 in. since the free flight aerodynamic database was Mach dependent, and experimental (wind tunnel) data existed for Mach 6 and Mach 10 conditions. Also shown in the figure are the uncertainty bounds, which were expanded at $X_{\mathrm{SEP}}=-44$ to better capture the uncertainty in the interference region aerodynamics. Similar increases were made in the uncertainty bounds for $\mathrm{C}_{\mathrm{A}}$ and $\mathrm{C}_{\mathrm{N}}$.

\section{Analysis Results \& Discussion}

\section{A. Redesign of Separation Control Logic}

Control of the research vehicle during separation was primarily concerned with managing the separation upset to avoid HXLV re-contact and large attitude excursions that would make it difficult to achieve the target attitude for the engine test. Because of the short $2.5 \mathrm{sec}$ duration of the separation event, the effectiveness of applying feedback control to improve separation performance is limited. Furthermore, the $\mathrm{X}-43 \mathrm{~A}$ control system was designed primarily to optimize vehicle performance during the engine test; thus, it was generally left only as a contingency option to change the gains and structure of the controller to improve separation performance. Consequently, for the Mach 7 flight, the separation control logic was largely a sequencer, defining when and how the various feedback loops of the control system were closed. In addition, there was also an elevator feed forward command. Considering the success of the Mach 7 separation as well as the constrained project timelines, this same structure of the separation control logic was retained for the Mach 10 flight.

The Mach 10 separation control logic structure consisted of two basic elements, namely loop closure times, and an elevator feed-forward command. Loop closure times specify when each of the feedback loops are closed including roll rate, yaw rate, pitch rate, angle of attack, and roll angle feedbacks. The yaw rate loop closure is unique in that it can be faded in over time. This fade is specified by two times, namely fade-start and fade-end. For the Mach 7 flight, the yaw rate fade was initiated $400 \mathrm{msec}$ after separation and ended at $1000 \mathrm{msec}$. The elevator feed-forward command is modeled as a linear function of separation angle of attack $\left(\mathrm{d}_{\mathrm{eff}}=\right.$ bias + slope* $\left.\mathrm{a}_{\text {sep }}\right)$. For the Mach 7 flight, the bias was set to 6 degrees and the slope was 0 . Although the Mach 7 structure was adopted, the flexibility 
did exist to adjust logic values. These adjustments were important in achieving predicted separation performance on par with the Mach 7 pre-flight predictions.

Re-design of the separation control logic was performed by parameterizing the structure described above and conducting a series of parametric studies and Monte Carlo analyses. The parameterization of the separation control logic resulted in the 8 design parameters: pitch rate loop (q-loop) closure time, roll rate loop (p-loop) closure time, yaw rate fade start time ( $r$-fade start), yaw rate fade end time (r-fade end), angle-of-attack loop ( $\alpha$-loop) closure time, roll angle loop ( $\phi$-loop) closure time, elevator feed-forward bias and elevator feed-forward slope.

A systematic approach was taken to determine the best combination of the above 8 parameters. Initially a benchmark Monte Carlo study was performed to establish a starting point and assess the significance of the problem. Next, parametric studies were conducted to identify promising regions of the trade space. At most, two parameters were varied at a time. Contour plots were used to identify regions of interest. Since these plots are based on a single test case (nominal or off-nominal), Monte Carlo analysis was used to assess statistical performance. Together, with the parametric study results it was possible to bound the potential values of each parameter. With ranges defined, additional time was afforded to explore candidate combinations. Again, more extensive parametric and Monte Carlo studies were used to ultimately select the nominal separation control logic settings.

1. Mach 10 Benchmark Monte Carlo

The benchmark Monte Carlo study consisted of 400 perturbed Mach 10 separation cases using the Mach 7 separation control logic parameters. These parameters included a 6 degree elevator feed-forward bias, zero slope, q-loop and p-loop closures at $100 \mathrm{msec}, \alpha$-loop and $\phi$-loop closures at $500 \mathrm{msec}$, a yaw rate fade-start at $400 \mathrm{msec}$, and a yaw rate fade-end at $1000 \mathrm{msec}$. Results of the study are shown in Table 2 and include a comparison to the final Mach 7 pre-flight Monte Carlo study.

Table 2. Mach 10 Benchmark Monte Carlo Results

\begin{tabular}{lcc}
\hline \hline & Mach 10 Benchmark & Mach 7 Monte Carlo \\
\hline Failures, \% & 6.3 & 2.4 \\
Re-contacts, \% & 0.3 & 0.9 \\
$\alpha-\beta$ Target Box, \% & 89.8 & 98.4 \\
Wing Rate Limiting, \% & 27.7 & 19.5 \\
Rudder Rate Limiting, \% & 8.3 & 1.5 \\
Wing Position Limiting, \% & $<1$ & $<1$ \\
Rudder Position Limiting, \% & 6.9 & $<1$ \\
\hline
\end{tabular}

The benchmark results show an overall degradation in separation performance at the conditions for the Mach 10 flight. With the Mach 7 control logic settings, the probability of being within the primary $\alpha-\beta$ target box $\left(0^{\circ}=\alpha=1^{\circ},-0.5^{\circ}=\beta=0.5^{\circ}\right)$ decreased, rudder rate and position limiting increased, and there was a higher overall failure rate. A case was considered failed if there was a re-contact, $\alpha$ or $\beta$ excursions exceeded $\pm 10 \mathrm{deg}$, or the RV attitude at the end of separation was outside minimum acceptable ranges $\left(0^{\circ}=\alpha=3^{\circ},-3^{\circ}=\beta=3^{\circ}\right)$. The only improvement with respect to the Mach 7 baseline was a decrease in the probability of a re-contact which occurred because of the favorable nose-down $\Delta \mathrm{C}_{\mathrm{m}}$ increment derived from CFD analysis and applied in the interference region to account for flight at Mach 10.

Some of the reduction in performance can be attributed to the effect of the increased Mach number on the vehicle aerodynamics. For instance, at Mach 10 the RV had less free flight stability margin and control effectiveness than at Mach 7. There were also performance losses due to model changes that were made in response to Mach 7 flight data reduction, such as the increased uncertainty bands around the aerodynamic interference coefficients and the extension of the aerodynamic interference region, which increased the time that the vehicle was affected by aerodynamic interference by roughly $150 \mathrm{~ms}$. Finally, changes in the RV and HXLV mass properties also had an effect on separation performance.

The objective of the separation control logic redesign was to recover as much of the lost separation performance as possible. As will be shown, much of this performance can be recovered by judicious selection of the separation control logic parameters. The failure rate can be reduced by adjusting the elevator bias. Additional reductions were achieved through adjustment of other separation control logic parameters. To a lesser extent, the rudder rate and position limiting could be reduced, but not to Flight 2 levels. Some of the mo deling differences between the Mach 7 and 10 simulations contribute to this discrepancy. 


\section{Parametric Studies}

Parametric studies were performed for two variables at a time. For the most part, a nominal case was used for parametrics involving longitudinal parameters (elevator feed-forward, $\alpha$-loop, and q-loop). An offnominal case was required to exercise the lateral-directional dynamics of the system and thus enable evaluation of the $\mathrm{p}$-loop, $\phi$ loop, and yaw rate fade times.

A key study involved variation of the elevator feed-forward bias along with the $\alpha$-loop closure. The other 6 control logic parameters were held at the benchmark values. Figure 7 is a typical contour plot produced in the parametric studies. For this case the color contours represent the rate change of angle of attack of the RV at 2.5 seconds and the black lines represent contours of constant angle of attack at $2.5 \mathrm{sec}-$ onds. The ideal combination is 0 rate and a value of 1.0 degree. An $\alpha$-loop in the neighborhood of $500 \mathrm{msec}$ provided low rates for entire elevator bias range. In order to refine the bounds on the bias, additional metrics were examined.

Two of the fundamental considerations in selecting the elevator bias were its impact on RV/HXLV proximity and wing rate limiting. Proximity is defined as the closest distance between the RV and HXLV at any point in the separation trajectory and is a measure of the re-contact potential. Higher values of elevator bias force larger pitch down moments on the RV which is good from a proximity standpoint. However, these larger moments cause additional stress on the vehicle control system in terms of its recovery. This is reflected in higher wing rates. On the other hand, if the elevator bias is too low, large wing rates are required to arrest the resulting pitch up response. Ultimately the choice of the bias must balance these considerations. The effect of the bias value on the wing rate is shown in Fig. 8 where the black lines on the contour plot define the range of elevator bias (roughly 2.5 to $5.5 \mathrm{deg}$ ) that limits peak wing rates to $\pm 50 \mathrm{deg} / \mathrm{s}$. Figure 9 shows the relationship between elevator bias and proximity. A spread in proximity of roughly 1 inch can be seen across the range of biases that were exa mined.

Another key parametric study was conducted to determine the yaw rate fade parameters. The tendency of the RV to rely on considerable rudder deflection in response to yawing motion has long been recognized. ${ }^{6}$ The large aerodynamic uncertainty associated with separation interference makes rudder activity particularly high during the separation event. In order to avoid the potential complications of rudder rate and position limiting the separation control logic allows the yaw rate feedback to be faded in over time. Effectively, design of the fade reduces to a trade between rudder activity and sideslip/roll angle excursions. 
For this study, an off-nominal case was chosen that had large rudder deflections and a peak $\beta$ of over $5 \mathrm{deg}$. This same case was run with a range of fade start times and duration. Figure 10 illustrates the results where color contours reflect maximum rudder position and the black traces are associated with contours of peak sideslip angle. Two regions of interest that have relatively low rudder activity are shaded. The first is associated with early start times and fade durations in the range of 300 to $800 \mathrm{msec}$. The second region is associated with later start times and moderate to high fade durations. The peak sideslip angle, and similarly roll angle (not pictured), varied predominantly with the fade start time, and was farily insensitive to the fade duration, Thus, lower fade start times led to lower values of $\beta_{\text {max. }}$. This explains why the fade start times associated with the second egion is limited to 500 to $600 \mathrm{msec}$. These two regions became the focus of additional Monte Carlo studies described below.

\section{Monte Carlo Studies}

Monte Carlo studies were used to compare the candidate settings related to the yaw rate fade. The two regions of interest previously identified in the yaw rate fade parametric study were targeted. Over 40 separate Monte Carlo analyses were run at a range of fade-start and duration settings within these regions. In addition, variation in the $\phi$-loop (100 and $500 \mathrm{msec})$ and elevator feed-forward bias (1 and 4 deg.) was included to capture potential coupling between logic parameters. The results showed that the two egions represented a choice between emphasizing lower failure rate vs. lower control surface activity. The results of a representative case from each region are shown in Table 3. By shifting the fade later in the separation timeline, the rudder rate and deflection limiting could be significantly decreased, but only with an accompanying degradation in the mission success statistics.

Monte Carlo studies were also used to quickly understand whether there was any advantage to delaying the closure of pitch and roll rate loops beyond the benchmark value of $100 \mathrm{msec}$. For both of these parameters, loop closure times less than $100 \mathrm{msec}$ were not considered because the RV does not leave the separation pistons until roughly that time. Two separate Monte Carlo studies were performed for both loops with closure times of $200 \mathrm{msec}$. In both cases separation performance degraded as loop closure times increased beyond $100 \mathrm{msec}$. Further Monte Carlo analysis was not performed since delaying rate feedback control any longer would only tend to further degrade performance.

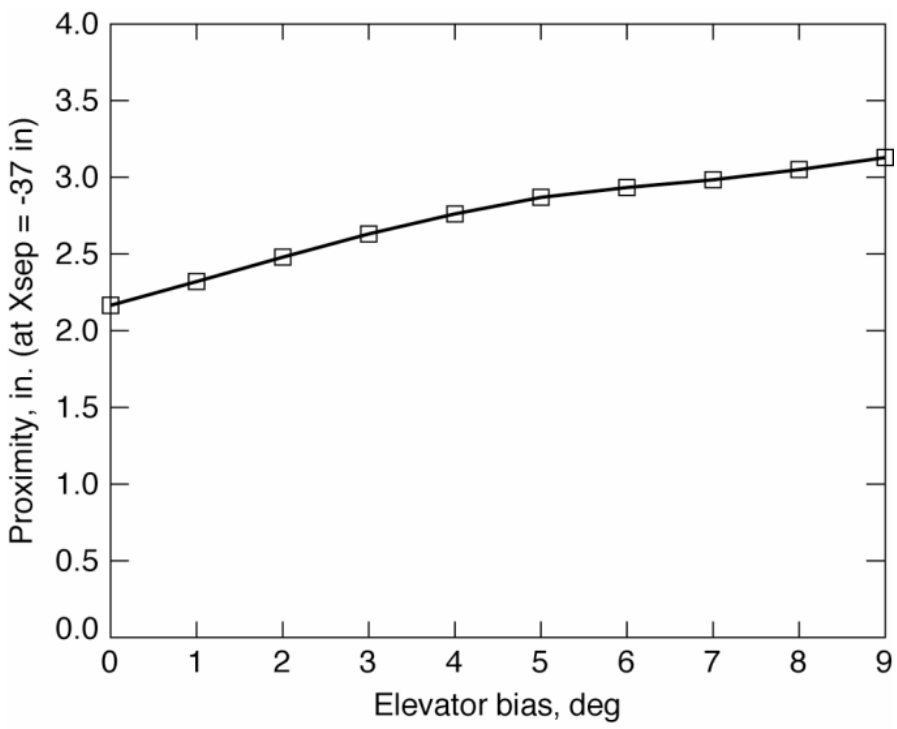

Figure 9. Effect of elevator bias setting on proximity at axial horizontal distance of 37 inches.

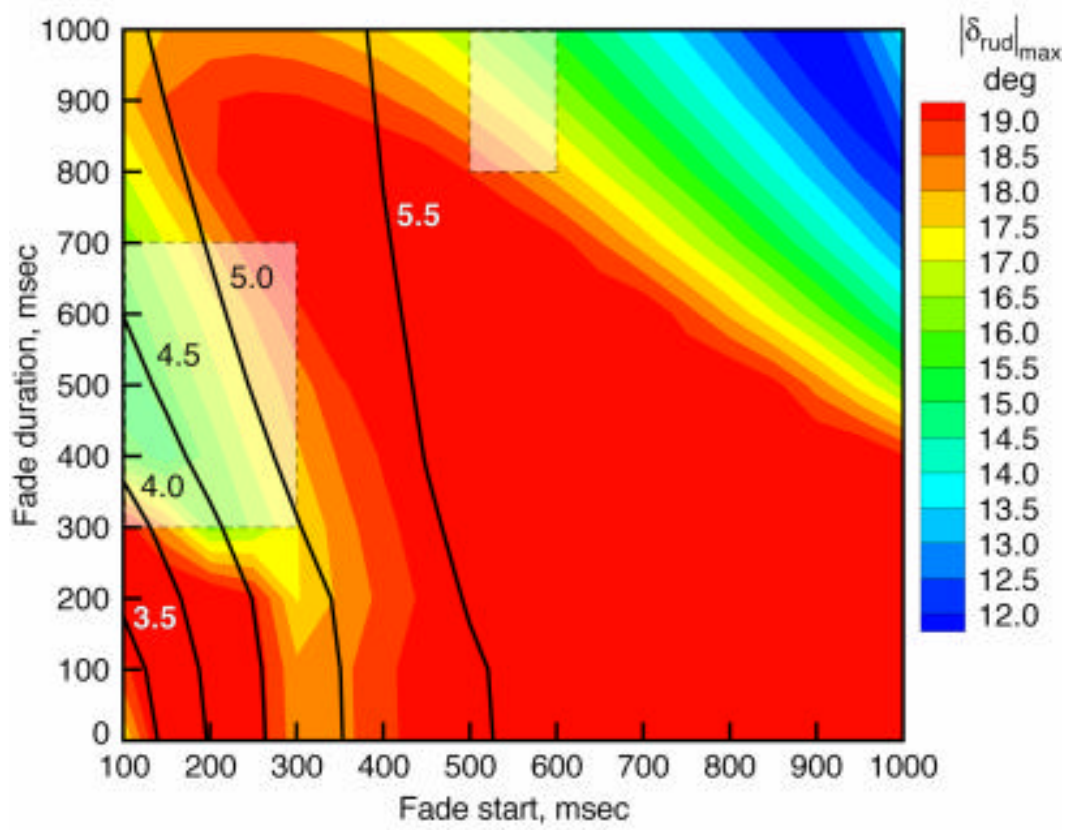

Figure 10. Parametric study results to identify promising regions of yaw fade parameter trade space.

American Institute of Aeronautics and Astronautics 
Table 3. Statistical Performance for Two Candidate Yaw Fade Strategies

\begin{tabular}{|c|c|c|}
\hline Metric & $\begin{array}{c}\text { Elev. bias }=3.5^{\circ} \\
\text { Fade } \text { start }=100 \mathrm{~ms} \\
\text { Fade end }=700 \mathrm{~ms} \\
(400 \text { cases })\end{array}$ & $\begin{array}{c}\text { Elev. bias }=3.5^{\circ} \\
\text { Fade start }=600 \mathrm{~ms} \\
\text { Fade end }=1500 \mathrm{~ms} \\
(400 \text { cases })\end{array}$ \\
\hline Failure Rate & $3.5 \%$ & $5.5 \%$ \\
\hline Within Primary $\alpha-\beta$ Target Box & $95.5 \%$ & $89.5 \%$ \\
\hline$\left|\beta_{\max }\right|>4 \operatorname{deg}$ & $5.2 \%$ & $8.2 \%$ \\
\hline Wing Rate > $100 \mathrm{deg} / \mathrm{s}$ & $23.1 \%$ & $21.7 \%$ \\
\hline Rudder Rate $>100 \mathrm{deg} / \mathrm{s}$ & $13.5 \%$ & $1.3 \%$ \\
\hline Reach Rudder Position Limits & $4.9 \%$ & $1.6 \%$ \\
\hline
\end{tabular}

\section{Mission Data Load}

Based on the results of these studies, a range of discrete values were defined for each of these parameters and included in the Mission Data Load (MDL). With the MDL the capability existed to upload selected flight control, navigation and propulsion parameters to the RV at mission time instead of early in the program when the flight software was released. The advantage of this arrangement is that parameters included in the MDL could be finalized much later in the project timeline; however, more MDL parameters meant that additional verification tests had to be run. For the Mach 10 flight, the separation logic parameters were added to the MDL, effectively removing further separation logic re-design from the critical path. However, the range of potential values had to be chosen carefully to limit the extent of verification testing needed to qualify the Mission Data Load of the Flight Management Unit. The ranges and nominal values are specified in Table 4 . The nominal slope and $\alpha$-loop closure times were selected from parametric study results. Monte Carlo comparisons were used to select the elevator bias, $\phi$-loop and yaw fade times. The statistical results of each Monte Carlo were combined in a cost function to determine which set of parameters performed best. The cost function was weighted to account for different objectives, with the final selection made using a cost function that weighted failure rate and target box accuracy five times more than surface rate and deflection limiting.

Table 4. Mission Data Load Ranges of Separation Control Parameters. Nominal Values in Red.

\begin{tabular}{|c|c|c|c|c|c|c|c|c|c|c|c|}
\hline Variable & & & & & & Values & & & & & \\
\hline Bias, deg & 1.0 & 1.5 & 2.0 & 2.5 & 3.0 & 3.5 & 4.0 & 4.5 & 5.0 & 5.5 & 6.0 \\
\hline Slope & -2 & -1 & $\mathbf{0}$ & 1 & & & & & & & \\
\hline q-loop closure, msec & 100 & & & & & & & & & & \\
\hline$\alpha$-loop closure, msec & 100 & 300 & 500 & 700 & & & & & & & \\
\hline p-loop closure, msec & 100 & & & & & & & & & & \\
\hline$\phi$-loop closure, msec & 100 & 300 & 500 & 700 & & & & & & & \\
\hline Start yaw-loop fade, msec & 100 & 200 & 400 & 500 & 600 & & & & & & \\
\hline End yaw-loop fade, msec & 500 & 600 & 700 & 800 & 900 & 1000 & 1300 & 1400 & 1500 & 1600 & \\
\hline
\end{tabular}

\section{B. Mach 10 Preflight Monte Carlo Analysis}

Table 5 compares the key results from the final pre-flight Mach 10 Monte Carlo analysis with the pre-flight Mach 7 values. Both sets of statistical results were derived from 1200 off-nominal cases. The results show an improvement over the Mach 10 benchmark case and demonstrate that much of the lost separation performance was recovered by adjusting the separation control logic settings. As expected, there were more failures and lower target box accuracy for the Mach 10 results when compared to the Mach 7 results. The number of failed cases increased primarily because there were more cases with $\alpha$ and $\beta$ excursions exceeding $10 \mathrm{deg}$. The biggest cause of the increase in large-excursion cases was the longer interference-to-free transition region since it required more extrapolation of the 8-dimensional interference aerodynamic database. Also, there were actually less re-contacts for the Mach 10 case, due primarily to the effect of the Mach 7-to-Mach 10 increments applied in the aerodynamic interference region which tended to increase the upward normal force and induce a more nose-down pitching moment on the RV. While the frequency of wing rate limiting increased only moderately in the Mach 10 results, there was significantly more rudder rate and position limiting than in the Mach 7 results. Some of this additional rudder activity was also 
Table 5. Final Mach 10 Pre-Flight Monte Carlo Results

\begin{tabular}{lcc}
\hline \hline & Mach 10 Baseline & Mach 7 Monte Carlo \\
\hline Number of Cases & 1200 & 1200 \\
Failures, \% & 4.4 & 2.2 \\
Re-contacts, \% & 0.2 & 0.9 \\
Primary Target Box, \% & 95.0 & 98.3 \\
Minimum $\alpha$ (average), deg & -1.64 & -1.40 \\
Peak $\beta$ (average +/-), deg & $1.12 /-0.80$ & $0.94 /-0.77$ \\
Wing Rate Limiting, \% & 24.5 & 19.5 \\
Rudder Rate Limiting, \% & 8.6 & 1.5 \\
Wing Position Limiting, \% & $<1$ & $<1$ \\
Rudder Position Limiting, \% & 4.4 & $<1$ \\
\hline
\end{tabular}

caused by the increased extrapolation of interference aerodynamic coefficients brought on by the longer transition region.

\section{C. $\mathbf{C m}_{\mathbf{0}}$ bias Stress Test}

A stress test was conducted with the Mach 10 simulation to ensure that the system was robust to the type of $\mathrm{Cm}_{0}$ bias error that was observed in the Mach 7 flight. First, two individual cases were run with the free flight pitching moment increment set to \pm 0.002 ; which was larger than the value that was deduced from the Mach 7 model reconciliation study. The resulting angle-of-attack profiles are shown in Fig. 11 and indicate that the RV is capable of recovering from either extreme in pitching moment bias. In addition, a Monte Carlo analysis was performed in which the free flight $\mathrm{Cm}_{0}$ increment was varied uniformly between -0.002 and +0.002 , and all other variables were varied using baseline uncertainty ranges. The results were similar to the results of the final pre-flight Monte Carlo, with only 2 additional failures in the $\mathrm{Cm} 0$ set. The resulting Monte Carlo uncertainty envelope for angle-ofattack is also shown in Fig. 11.

\section{Mach 10 Flight Performance}

On Nov. 16, 2004 the final X43A flight occurred with a fully successful stage separation. ${ }^{11-12}$ The HXLV accurately delivered the RV to the separation conditions well within one standard deviation of the target values. The RV exhibited nearly nominal performance during separation and based on analysis of flight data there was no indication of a re-contact. Figure 12 shows the angle-of-attack profile reconstructed from flight telemetry as well as the predicted profile from the simulation (with flight-derived initial conditions). As in the Mach 7 flight, the RV took longer than predicted to reach the commanded angle-of-attack, again, most likely

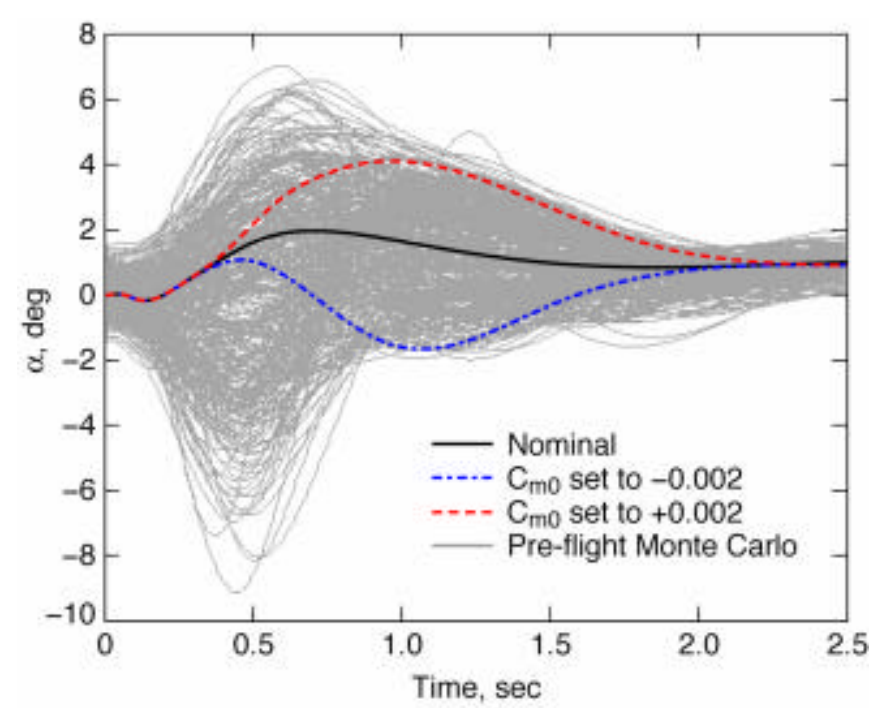

Figure 11. Results of Mach $10 \mathrm{Cm}_{0}$ bias stress test.

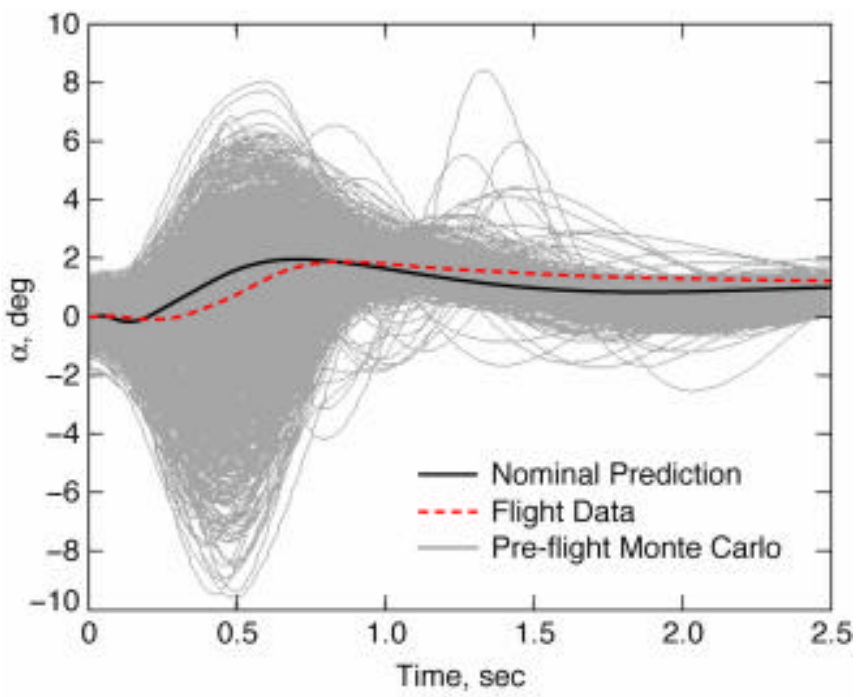

Figure 12. Comparison of Mach $10 \mathrm{RV}$ angle-of-attack time history. Flight data vs. pre-flight Monte Carlo. 
due to inaccuracies in the prediction of the pitch trim location. Even so, the RV still achieved the required angle-of-attack prior to the engine test, and did so quicker than in the Mach 7 flight because of the controller modification that increased the angleof-attack integrator gain. Finally, note that the entire angle-of-attack response was captured within the pre-flight Monte Carlo uncertainty bound.

Figure 13 shows the elevator deflection during separation for the Mach 10 flight. Again, the flight data was well captured within the pre-flight Monte Carlo envelope. The small bias after the transient is due to the error in $\mathrm{Cm} 0$, resulting from the incorrect trim location in the simulation aerodynamic database. There was very little lateraldirectional $\mathbf{e}$ sponse during the Mach 10 separation, indicating that both pistons fired nearly simultaneously. The sideslip angle time history and pre-flight Monte Carlo bounds are shown in Fig. 14.

\section{Conclusion}

The success of stage separation during the first powered flight of the X-43A in March 2004 provided confidence in the separation analysis approach and simulation modeling. For the next flight conducted at Mach 10 conditions, a similar approach was undertaken and the same simulation structure was adopted. Considerable effort was made to identify Mach 10 model updates through Mach 7 flight data reduction, model reconciliation studies, and CFD analysis. Once these updates were made, benchmark analysis of the Mach 10 mission showed degraded performance relative to Mach 7 pre-flight predictions. Much of the performance was recovered by adjusting the feed-forward elevator command and feedback loop closure times. This redesign of the separation control logic was achieved through extensive parametric studies and Monte Carlo analyses conducted using POST. In addition, an independent, high-fidelity ADAMS simulation was also maintained throughout the analysis cycle to understand the effects of high-order models and to verify the POST simulation. Numerous Monte Carlo trade studies with conservative uncertainty models were performed with these tools to understand the impact of modeling assumptions and to perform stress testing to ensure that the system was robust to anomalies observed in the Mach 7 flight.

On November 16, 2004 the X-43A Mach 10 flight occurred with another successful stage separation. The Research Vehicle exhibited near-nominal performance throughout separation and the vehicle performed within the uncertainty bounds predicted in the pre-flight analysis and went on to break the world record for airbreathing powered flight, set previously by the Mach 7 X-43A flight.

\section{Acknowledgments}

The authors would like to thank Anne Rhodes for her work in preparing this manuscript for publication. 


\section{References}

${ }^{1}$ Marshall, L. A., Corpening, G. P., and Sherrill, R., “A Chief Engineer's View of the NASA X-43A Scramjet Flight Test,” American Institute of Aeronautics and Astronautics, AIAA Paper 2005-3332, May 2005.

${ }^{2}$ Bahm, C., Baumann, E., Martin, J., Bose, D., Beck, R. E., and Strovers, B., "The X-43A Hyper-X Mach 7 Flight 2 Guidance, Navigation, and Control Overview and Flight Test Results," American Institute of Aeronautics and Astronautics, AIAA Paper 2005-3275, May 2005.

${ }^{3}$ Anon.: Using ADAMS/Solver. Users Guide, Vol. 9.0.1, Mechanical Dynamics, Inc.

${ }^{4}$ Brauer, G. L., Cornick, D. E., and Stevenson, R., "Capabilities and Applications of the Program to Optimize Simulated Trajectories (POST)," NASA CR-2770, Feb. 1977.

${ }^{5}$ Powell, R. W., et. al., "Program to Optimize Simulated Trajectories (POST II) Utilization Manual, Version 1.1.7," July 2002.

${ }^{6}$ Tartabini, P. V., Bose, D. M., McMinn, J. D., Martin, J. G., and Strovers, B. K., "Hyper-X Stage Separation Trajectory Validation Studies," American Institute of Aeronautics and Astronautics, AIAA Paper 2003-5819, August 2003.

${ }^{7}$ Blocker, W. D. and Reubush, D. E., "X-43A Stage Separation System - A Flight Data Evaluation," American Institute of Aeronautics and Astronautics, AIAA Paper 2005-3335, May 2005.

${ }^{8}$ Engelund, W. C., Holland, S. D., Cockrell, C. E. and Bittner, R. D., "Aerodynamic Database Development for the Hyper-X Airframe-Integrated Scramjet Propulsion Experiments,” Journal of Spacecraft and Rockets, Vol. 38, No. 6, 2001, pp. 803-810.

${ }^{9}$ Woods, W. C., Holland, S. D., and DiFulvio, M., "Hyper-X Stage Separation Wind-Tunnel Test Program," Journal of Spacecraft and Rockets, Vol. 38, No. 6, 2001, pp. 811-819.

${ }^{10}$ Buning, P. G., Wong, T. C., Dilley, A. D., and Pao, J. L., "Computational Fluid Dynamics Prediction of Hyper-X Stage Separation Aerodynamics," Journal of Spacecraft and Rockets, Vol. 38, No. 6, 2001, pp. 820-827.

${ }^{11}$ Marshall, L. A., Bahm, C., Corpening, G. P., and Sherrill, R., "Overview With Results and Lessons Learned of the X-43A Mach 10 Flight," American Institute of Aeronautics and Astronautics, AIAA Paper 2005-3336, May 2005.

${ }^{12}$ Karlgaard, C. D., Martin, J. G., Tartabini, P. V., and Thornblom, M. N., "Hyper-X Mach 10 Trajectory Reconstruction," American Institute of Aeronautics and Astronautics, AIAA Paper 2005-5920, August 2005. 